Neuroscience

\title{
Stimulus selectivity of single neurons in the temporal lobe
}

\section{Michael Rugg}

THE crucial contribution to memory made by the structures of the medial temporal lobe of the brain' - the hippocampus, amygdala and adjacent cortical regions (see figure) - has been investigated by examining the effects on learning and memory of damage to these structures. A complementary approach to elucidating the functions of medial temporal-lobe structures, that of recording from single neurons during behaviour, has been systematically applied only in non-human species. An advance towards bridging this gap has now been made by Heit et al., who on page 773 of this issue $^{2}$ describe the activity of single neurons in the medial temporal lobes of epileptic patients in whom intracerebral electrodes have been implanted for clinical reasons. A high proportion of these neurons displays remarkable selectivity for individual members of a repeatedly presented set of 10 words. While one cell shows enhanced firing each time the word 'luck' is presented, for

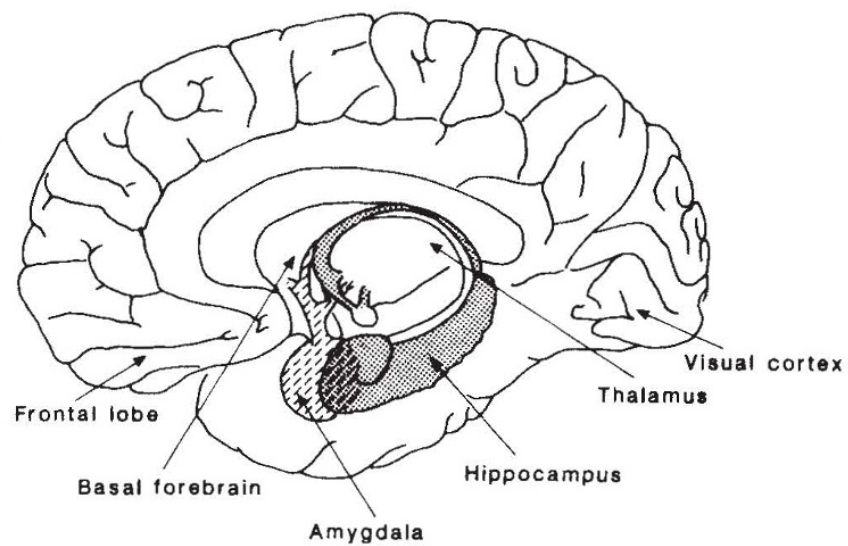

Section of the human brain showing the relation of the structures of the medial temporal lobe to the cortex and sub-cortical structures. stimulus) has been encountered in the recent past - but do not interfere with the comprehension and usage of words in everyday language. It therefore seems likely, at least with familiar stimuli such as words, that structures within the medial temporal lobe contribute to memories about the specific episodes in which a stimulus is encountered, rather than about aspects of the stimulus that remain con- recorded averaged evoked potentials do change with stimulus repetition, indicating that some type of neuronal activity within the medial temporal lobe discriminates repeated from unrepeated stimuli. This activity could be related to efferent information, unavailable to the cells from which unit recordings were obtained by Heit et al., which signal the occurrence of stimulus repetition to other brain regions.

Another way of viewing the data of Heit et al. is to compare the cells they describe with the 'place' cells in the hippocampus of the rat. Place cells are so named because of their sensitivity to the animal's location in its environment. They fire selectively each time the same spatial location is entered, and may be part of a parallel distributed system representing example, another responds preferentially to 'carve'.

A striking feature of these data is the frequency with which word-specific responses were observed; 75 per cent of the neurons from which recordings could reliably be obtained show selectivity for at least one of the 10 words in the list tested. In view of the restricted nature of this list, such a high rate of success in finding wordselective cells implies that each word must have actually evoked activity in many neurons. Together with the fact that some cells are responsive to more than one word, this suggests that words are represented in this neural population by virtue of the pattern of activity they evoke. Such a form of stimulus representation is one of the cornerstones of the "parallel distributed' models of brain function that are currently under intensive development

The findings of Heit et al. therefore provide support for the view that complex stimuli such as words are subject to some form of population coding in the human brain, but the relevance of these data to understanding the role played by the medial temporal lobe in learning and memory is less clear. Bilateral lesions of this region severely impair recognition memory - the ability to remember whether a particular word (or any other stant across different episodes, s
word's meaning or pronunciation.

This being so, it is perhaps surprising that Heit et al. find that their cells show the same level of firing when the preferred word is shown on the first occasion as when it is repeated, implying that these cells do not encode the information necessary to recognize the re-occurrence of the word. The interpretation of this finding favoured by Heit et al. is that the output of the medial temporal lobe to other brain regions does not change as a consequence of stimulus repetition. But this conclusion can be questioned on several grounds.

First, recordings from the hippocampus of macaque monkeys reveal changes in neuronal activity dependent on the context in which a stimulus is presented, or that occur as a consequence of stimulus repetition ${ }^{4,5}$. Thus, in a species closely related to man, there is evidence that some hippocampal neurons do show memoryrelated changes in their firing rates.

Second, before concluding that the human medial temporal lobe does not change its output when stimulus repetition occurs, it is necessary to demonstrate that the cells sampled actually include ones that reflect its efferent activity. This has yet to be achieved.

Third, Heit et al. report that locally the current spatial environment ${ }^{6}$. In 1978, O'Keefe and $\mathrm{Nadel}^{7}$ suggested that the hippocampus of higher species represents non-spatial information in an analogous fashion, forming so-called cognitive maps of, for example, the semantic attributes of words. The data of Heit et al. could provide evidence for such nonspatial representations in the human hippocampus and adjacent structures. For the reasons noted above, such representations are unlikely to be necessary for the retrieval of attributes of a stimulus such as its meaning, but may be used in forming associations between a stimulus and the context in which it is presented.

A final interesting feature of the findings of Heit et al. concerns the diversity of the structures in which they find "wordspecific' neurons, including the hippocampus and the amygdala, two structures which make separate contributions to memory, as shown by lesion studies in primates The existence of stimulus-specific neurons with the same properties in these two structures suggests that, although they perform different functions, the hippocampus and amygdala use a common means of representing a stimulus undergoing processing. Whether other brain regions possess neuronal populations that represent complex stimuli in a similar way is an interesting question for the future. $\square$

1. Scoville. W.B. \& Milner, B. J. neurol. Neurosurg. Psychiat. 20. 11-21 (1957)

Heit. G. et al. Nature 333. 773-775 (1988)

McClelland. J.L. \& Rumelhart. D.E. (eds) Parallel Dis. tributed Processing: Explorations in the Microstructure of Cognition (MIT Cambridge, Massachusetts, 1986)

4. Brown. M.W. in Neuronal Plasticity and Memory Formation (eds Ajmone Marsan. C. \& Matthies. H.) 557-573 (Raven. New York. 1982).

Rolls. E.T. et ul. Soc: Neurosci. Abstr. 11. 525 (1985).

O'Keefe. J. \& Speakman. A. Expl Brain Res. 68. 1-27 (1987)

. O'Keefe, J. \& Nadel. L. The Hippocampus as a Cognitive Map (Oxford University Press. 1978)

Mishkin. M Phil Trans. R. Soc. B298, 85-95 (1982)

. Mishkin. M. \& Appenzeller. T. Scient. Am. 255, 80 (1987).

Michael Rugg is in the Department of Psychology, University of St Andrews, KY16 9JU, UK. 